\title{
Cambios en el consumo aparente de lácteos en hogares con niños menores de 19 años en el Paraguay en el periodo 1997 a 2012
}

\author{
Changes in the apparent consumption of dairy products in \\ households with children under 19 years of age in Paraguay \\ from 1997 to 2012
}

\author{
Vit Bubak ${ }^{1}$, Leticia Ramírez Pastore ${ }^{2}$, Marta Sanabria ${ }^{2}$
}

\section{RESUMEN}

Introducción: Los productos lácteos contribuyen significativamente a la ingesta de nutrientes de la población; por lo tanto, entender los cambios en consumo de productos lácteos representa una parte importante del sistema de vigilancia nutricional. Objetivo: Describir los cambios en los gastos relativos en el consumo aparente de productos lácteos (con y sin auto-producción) y el consumo aparente de calcio en hogares paraguayos con niños menores de 19 años durante el período 1997 a 2012. Materiales y Métodos: Un análisis descriptivo-analítico de muestras representativas de hogares de la Encuesta Integrada de Hogares (EIH 1997-98) y la Encuesta de Ingresos y Gastos (EIG 2011-12), llevadas a cabo por la Oficina de Estadística, Encuestas y Censos (DGEEC). El análisis se realizó para todos los hogares encuestados, tanto por quintiles de ingresos (Q1-Q5) como por área de residencia (rural/urbana). Resultados: Tanto los gastos relativos como el consumo aparente (CA) de productos lácteos (incluyendo la auto-producción) aumentaron mínimamente entre 1997 y 2012: los gastos relativos aumentaron del $12,8 \%$ al $13 \%$ y el CA aumentó de $431 \mathrm{cc} \mathrm{a}$ 437 cc per cápita/día. En términos de productos lácteos individuales, el CA de leche y el CA de leche en polvo disminuyeron $(-7.6 \%$ y $-47.5 \%)$, mientras que el CA de queso y el CA de yogur y otros productos lácteos aumentaron ( $26 \%$ y $67.1 \%)$. El CA de productos lácteos exhibió cambios diferenciales según el ingreso del hogar, disminuyendo en un $3.1 \%$ en los hogares con los ingresos más bajos (Q1) y aumentando en un 6.5\% en los hogares con los ingresos más altos (Q5). Del mismo modo, el CA de calcio aumentó marginalmente entre 1997-2012 (de 502 mg a $514 \mathrm{mg}$ per cápita/día), disminuyendo en un 2,6\% en los

\section{ABSTRACT}

Introduction: Dairy products contribute significantly to the nutrient intake of the population; therefore, understanding the changes in dairy consumption is an important aspect of the nutritional surveillance system. Objective: To describe the changes in relative expenditures related to the apparent consumption of dairy products (with and without self-production) and the apparent consumption of calcium in Paraguayan households with children under 19 years of age during the 1997 to 2012 time period. Materials and Methods: This was a descriptive-analytical study of representative samples of households from the Integrated Household Survey (EIH 1997-98) and the Income and Expenditure Survey (EIG 2011-12), carried out by the Office of Statistics, Surveys and Censuses (DGEEC). The analysis was carried out for all surveyed households, both by income quintiles (Q1-Q5) and by area of residence (rural / urban). Results: Both relative expenditures and apparent consumption (AC) of dairy products (including self-production) increased minimally between 1997 and 2012: relative expenses increased from $12.8 \%$ to $13 \%$ and AC increased from 431 cc to 437 cc per capita per day. In terms of individual dairy products, the AC of milk and the AC of powdered milk decreased $(-7.6 \%$ and $-47.5 \%)$, while the $\mathrm{AC}$ of cheese and the AC of yogurt and other dairy products increased $(26 \%$ and $67.1 \%)$. The AC of dairy products showed differential changes according to household income, decreasing by $3.1 \%$ in households with the lowest income $(\mathrm{Q} 1)$ and increasing by $6.5 \%$ in households with the highest income (Q5). Similarly, calcium AC increased marginally between 1997-2012 (from $502 \mathrm{mg}$ to $514 \mathrm{mg}$ per capita per day), decreasing by

\footnotetext{
${ }^{1}$ Instituto Desarrollo. Asunción. Paraguay.

${ }^{2}$ Universidad Nacional de Asunción. Hospital de Clínicas. Cátedra y Servicio de Pediatría. San Lorenzo. Paraguay.

Correspondencia: Marta Cristina Sanabria Correo: marta.sanabria@gmail.com

Conflicto de interés: Los autores declaran no poseer conflicto de interés

Recibido: 17/04/2018 Aceptado: 17/07/2018

DOI: https://doi.org/10.31698/ped.45022018003
} 
hogares con los ingresos más bajos (Q1) y aumentando en un 7,2\% en los hogares con los ingresos más altos (Q5). Tanto en 1997 como en 2012, el CA de productos lácteos y de calcio aumentó con el ingreso del hogar, pero solo los hogares de mayores ingresos (Q5) alcanzaron las recomendaciones diarias de productos lácteos y calcio. La auto-producción contribuyó de manera importante a mejorar la ingesta diaria recomendada de productos lácteos y calcio, especialmente en las zonas rurales. Conclusiones: El consumo aparente de productos lácteos y de calcio en los hogares paraguayos con niños menores de 19 años cae por debajo de las cantidades diarias recomendadas para todos los hogares con la excepción de los hogares de mayores ingresos.

Palabras clave: Calcio, leche, productos lácteos.
$2.6 \%$ in households with the lowest income (Q1) and increasing by $7.2 \%$ in households with the highest income (Q5). In both 1997 and 2012, the AC of dairy products and calcium increased with household income, but only the highest-income households (Q5) reached the daily recommendations for dairy products and calcium. Selfproduction contributed significantly to improving the recommended daily intake of dairy products and calcium, especially in rural areas. Conclusions: The apparent consumption of dairy products and calcium in Paraguayan households with children under 19 falls below the recommended daily allowances for all households with the exception of higher-income households.

Keywords: Calcium, milk, dairy products.

\section{INTRODUCCIÓN}

Los cambios en la alimentación, los patrones de trabajo y el tiempo libre conocidos a menudo como la "transición nutricional" contribuyen junto con los factores causales subyacentes a las enfermedades no transmisibles ${ }^{(1)}$. Por tanto, comprender los cambios en la dieta que caracterizan esta transición es clave para un diseño de estrategias alimentarias efectivas.

Los productos lácteos constituyen un grupo de alimentos cuyo consumo está experimentando cambios significativos como parte del proceso de transición nutricional. Comprender estos cambios representa, por lo tanto, un objetivo importante del sistema de vigilancia nutricional ${ }^{(2)}$.

Los productos lácteos contribuyen significativamente a la ingesta de nutrientes de la población, especialmente cuando se trata de ingesta de vitamina D, calcio, potasio, proteína, vitamina A, vitamina B12, fósforo, zinc y riboflavina ${ }^{(3)}$ De hecho, proporcionan más calcio, proteínas, magnesio, potasio, zinc y fósforo por caloría que cualquier otro alimento típico que se encuentre en la dieta de los adultos ${ }^{(4)}$. La biodisponibilidad de algunos nutrientes en la leche (incluido el calcio) es alta en comparación con la de otros alimentos en la dieta ${ }^{(5)}$.

La leche y los productos lácteos también pueden ser importantes para diversificar la dieta. Son ricos en nutrientes y proporcionan proteínas y micronutrientes de alta calidad en una forma de fácil absorción que puede beneficiar tanto a las personas nutricionalmente vulnerables como a las personas sanas cuando se consumen en cantidades apropiadas. Los productos alimenticios a base de leche también se han utilizado con éxito en el tratamiento de la malnutrición moderada y grave en los niños. La leche entera en su forma en polvo enriquecida con hierro, calcio, zinc, cobre y vitamina $C$ es un componente clave del Programa Alimentario Nutricional Integral (PANI), programa dirigido a niños menores de 5 años que están desnutridos o en riesgo de desnutrición.

Este estudio describe los cambios en el consumo aparente de productos lácteos (con y sin autoproducción) en los hogares paraguayos con niños menores de 19 años durante el período entre 1997 y 2012, utilizando datos de la Encuesta Permanente de Hogares (EPH) 1997/98 y la Encuesta de Ingresos y Gastos (EIG) de 2011-12. El estudio evalúa tanto el consumo absoluto de productos lácteos (expresado en cc/per cápita/día) como el de calcio (expresado en $\mathrm{mg} /$ per cápita/día) en relación con las pautas dietéticas nacionales y los valores recomendados de referencia dietética para el calcio. Como parte inicial del análisis se evaluaron los cambios en los gastos alimentarios absolutos y relativos durante el período de estudio.

El análisis se realizó tanto para la muestra en su 
conjunto como para el área rural/urbana y los quintiles de ingreso. La metodología empleada en este estudio fue utilizada y validada por Crovetto y Uauy en estudios similares ${ }^{(6)}$.

\section{MATERIALES Y MÉTODOS}

Se utilizó los datos recopilados de la Encuesta Integrada de Hogares (EIH) de 1997-98 y de la Encuesta de Ingresos y Gastos (EIG) de 2011-12. Ambas encuestas fueron implementadas por la Dirección General de Estadística, Encuestas y Censos (DGEEC). La encuesta EIH 1997-98 se implementó entre agosto de 1997 y agosto de 1998 y abarcó un total de 4.353 hogares, la encuesta EIG 2011-12 se implementó entre agosto 2011 y julio de 2012 y cubrió un total de 5.417 hogares. Ambas encuestas son representativas a nivel nacional y subnacional y contienen módulos sobre los ingresos de los hogares y los gastos de alimentos, entre otros.

Tanto la encuesta EIH 1997-98 como EIG 2011-12 proporcionan información detallada sobre la cantidad y el gasto correspondiente a cada artículo alimenticio específico (o grupo de artículos) comprados u obtenidos de otro modo por el hogar durante los últimos 7 días.Con fines comparativos, las cantidades y los gastos en alimentos comprados (u obtenidos de otro modo) con frecuencia diaria (por ejemplo, todos los días, días alternos, 2, 3 y 4 veces por semana, o una vez a la semana) se convirtieron en sus equivalentes semanales usando un factor de conversión apropiado; las cantidades y los gastos en alimentos comprados (u obtenidos de otro modo) con una frecuencia inferior a la semanal no se modificaron, ya que los datos EIH 1997-98 no brindan esta información.

Con el fin de analizar los gastos en alimentos, se clasificó los alimentos en 13 grupos generales de alimentos, incluidos 1) cereales, 2) verduras, 3) frutas, 4) carne, 5) huevos, 6) productos lácteos, 7) aceites y grasas, 8) dulces, 9) bebidas no alcohólicas, $10)$ bebidas alcohólicas, 11) especias y condimentos, 12) otros alimentos (no incluidos en otros grupos), $y$ 13) alimentos que se consumen fuera del hogar. El grupo de "cereales" incluye tortas, galletas, panes, pasta, maíz, avena, arroz y otros cereales; el grupo de "verduras" incluye verduras de color naranja / rojo y verde oscuro, legumbres, vegetales con almidón y otras verduras; el grupo de "carnes" incluye carnes, aves, mariscos y carne de órganos; los "productos lácteos " incluyen leche fresca y pasteurizada, leche en polvo, queso y yogur y otros productos lácteos (excepto mantequilla); el grupo de 'aceites y grasas' incluye aceite, grasa, manteca y manteca (de leche), entre otros. Finalmente, los alimentos que se consumen fuera del hogar incluyen comidas preparadas y alimentos que se consumen fuera del hogar.

Los gastos en alimentos y el ingreso familiar se expresaron en términos reales utilizando los precios constantes de diciembre de 2011. Para este fin, se el índice de precios al consumidor general (IPC) para ajustar los ingresos del hogar y el IPC para alimentos (un componente del IPC general) para ajustar los precios de los alimentos. Dado que entre julio de 1997 y julio de 1998, los precios medios (de alimentos) aumentaron en 11.5 (11.8) por ciento, se utilizó el IPC promedio (IPC de alimentos) entre julio de 1997 y julio de 1998 al calcular el ajuste.

La muestra inicial de la encuesta EIH 1997-98 tuvo un total de 4.353 hogares y 20.487 miembros del hogar. De estos, un total de 3.444 hogares (79.1\%) tuvieron niños menores de 19 años. Hubo un total de 10.194 niños menores de 19 años. La edad promedio de estos niños fue de 8.58 años (DE 5.27) y la mediana de edad fue de 8 años. Al centrarse en los hogares con información completa sobre gastos de alimentos se restringió la muestra a 3.405 hogares.

La muestra inicial de la encuesta EIG 2011-12 tuvo un total de 5.417 hogares y 21.130 miembros del hogar. De estos, un total de 3.738 hogares (69.0\%) tuvieron niños menores de 19 años. Hubo un total de 8.480 niños menores de 19 años. La edad promedio de estos niños fue de 9.43 años (DE 5.40) y la mediana de edad fue de 10 años. Al centrarse en los hogares con información completa sobre gastos de alimentos se restringió la muestra a 3.720 hogares. De los 8.480 niños, poco más de la mitad (52.4 vivía en los $40 \%$ de hogares más pobres según el quintil de ingreso $(28.5 \%$ en Q1 y 23.8\% en Q2). El 47.6\% restante vivía en los $60 \%$ de hogares más ricos según el quintil de ingreso ( $18.4 \%$ in Q3, en Q3, $16.0 \%$ en Q4, y 13.1\% en Q5).

En el análisis de los gastos en alimentos, solo se 
consideraron los alimentos comprados por el hogar. No se consideraron artículos de comida recibidos como un regalo, de la iglesia, o como un beneficio de los programas de nutrición o sociales. Además, aunque no se consideraron los alimentos de producción propia en el análisis de los gastos de los hogares, sí se consideró la autoproducción (o el autoconsumo) en el análisis del consumo aparente de productos lácteos y calcio.

Una acotación final se refiere a la conversión de la cantidad de productos lácteos comprados de gramos (o kilogramos) a mililitros (más adelante referidos como centímetros cúbicos o cc). Para convertir gramos de queso en mililitros, se siguió las Nuevas Guías Alimentarias del Paraguay ${ }^{(7)}$ que establecen que una pieza de queso de $30 \mathrm{~g}$ equivale aproximadamente a una taza de $200 \mathrm{ml}$ de leche fluida entera. Cuando es necesario, se convirtió gramos de leche en mililitros de leche suponiendo una densidad promedio de leche de $1.031 \mathrm{~g} / \mathrm{cm}^{3}$. Para convertir la leche en leche en polvo, se empleó un factor de conversión del 10 por ciento (es decir, $100 \mathrm{~g}$ de leche en polvo producen $1.000 \mathrm{ml}$ de leche líquida).
Finalmente, para obtener los niveles de calcio correspondientes al consumo aparente de productos lácteos, se supuso un contenido promedio de $120 \mathrm{mg}$ de calcio por $100 \mathrm{ml}$ de leche, $90 \mathrm{mg}$ de calcio por 100 $\mathrm{ml}$ de leche en polvo reconstituida, $750 \mathrm{mg}$ de calcio por $100 \mathrm{~g}$ de queso, y $125 \mathrm{mg}$ de calcio por $100 \mathrm{~g}$ de yogur y otros productos lácteos.

\section{RESULTADOS}

La Tabla 1 resume la evolución de los gastos relativos de alimentos en los hogares en los grupos de alimentos con respecto al gasto total en alimentos, tanto para el todos los hogares (TH) como para los quintiles de ingreso (Q1 a Q5). El cuadro muestra que, en el período 2011-12, el grupo de 'leche y productos lácteos' representó la tercera mayor proporción de gastos relativos de alimentos de los hogares paraguayos $(13 \%)$, precedido solo por grupo de 'carne' $(29,6 \%)$ y el grupo de 'cereales' $(16.9 \%)$. Juntos, estos tres grupos de alimentos representaron casi el $60 \%$ del gasto total en alimentos de los hogares en 2011-12.

Tabla 1. Gastos relativos en grupos de alimentos para todos los hogares y por quintiles de ingresos (en \%)

\begin{tabular}{|c|c|c|c|c|c|c|c|c|c|c|c|c|}
\hline \multirow[t]{2}{*}{ Grupo de alimentos } & \multicolumn{2}{|c|}{ TH } & \multicolumn{2}{|c|}{ Q1 } & \multicolumn{2}{|c|}{ Q2 } & \multicolumn{2}{|c|}{ Q3 } & \multicolumn{2}{|c|}{ Q4 } & \multicolumn{2}{|c|}{ Q5 } \\
\hline & $1997-98$ & $2011-12$ & 1997-98 & $2011-12$ & 1997-98 & $2011-12$ & $1997-98$ & $2011-12$ & 1997-98 & 2011-12 & 1997-98 & $2011-12$ \\
\hline Cereales & 16.6 & 16.9 & 20.1 & 23.6 & 18.7 & 18.7 & 17.3 & 17.4 & 15.4 & 15.5 & 14.1 & 13.0 \\
\hline Hortalizas & 13.5 & 10.7 & 14.1 & 11.4 & 13.3 & 11.2 & 14.1 & 11.0 & 13.3 & 10.7 & 13.3 & 9.3 \\
\hline Frutas & 5.3 & 4.3 & 3.6 & 2.5 & 3.9 & 3.4 & 4.6 & 4.2 & 5.9 & 4.8 & 7.2 & 5.5 \\
\hline Carne & 27.3 & 29.6 & 27.1 & 27.7 & 27.9 & 30.4 & 27.7 & 30.0 & 27.3 & 29.7 & 26.9 & 29.4 \\
\hline Huevos & 2.0 & 1.4 & 2.2 & 1.1 & 1.9 & 1.4 & 2.2 & 1.6 & 2.0 & 1.6 & 2.0 & 1.3 \\
\hline Leche y productos lácteos & 12.8 & 13.0 & 11.4 & 9.4 & 12.3 & 11.7 & 12.4 & 12.6 & 12.8 & 14.1 & 14.3 & 14.9 \\
\hline Aceites y grasas & 4.5 & 3.5 & 6.2 & 6.1 & 5.8 & 4.0 & 4.9 & 3.2 & 3.6 & 2.9 & 3.1 & 2.6 \\
\hline Dulces & 3.8 & 5.0 & 4.3 & 6.1 & 4.3 & 5.1 & 3.7 & 5.0 & 3.7 & 4.7 & 3.5 & 4.7 \\
\hline Bebidas no alcoholicas & 7.6 & 7.9 & 6.7 & 6.7 & 7.2 & 7.2 & 7.6 & 7.6 & 7.8 & 8.2 & 8.3 & 8.9 \\
\hline Bebidas alcohólicas & 2.4 & 2.1 & 1.4 & 0.9 & 1.6 & 1.8 & 2.0 & 2.0 & 3.3 & 2.2 & 2.8 & 2.7 \\
\hline Especias y condimentos & 1.5 & 1.3 & 1.4 & 1.3 & 1.5 & 1.3 & 1.5 & 1.2 & 1.5 & 1.2 & 1.5 & 1.3 \\
\hline Otros alimentos & 0.5 & 1.3 & 0.3 & 1.6 & 0.4 & 1.3 & 0.4 & 1.1 & 0.6 & 1.2 & 0.5 & 1.3 \\
\hline \multirow[t]{2}{*}{ Comidas preparadas y FDH } & 2.0 & 3.3 & 1.2 & 1.5 & 1.3 & 2.5 & 1.6 & 3.0 & 2.9 & 3.1 & 2.5 & 4.9 \\
\hline & 100.0 & 100.0 & 100.0 & 100.0 & 100.0 & 100.0 & 100.0 & 100.0 & 100.0 & 100.0 & 100.0 & 100.0 \\
\hline
\end{tabular}

La Tabla 2 resume los cambios porcentuales en los gastos relativos en grupos de alimentos con respecto al gasto total en alimentos durante los períodos 1997-98 y 2011-12; estos se derivan de los valores porcentuales en la Tabla 3. Se puede observar una disminución importante en los gastos relativos de alimentos en 'frutas', 'vegetales' y 'huevos' (entre otros) y un aumento en los gastos relativos de alimentos en 'carnes', tanto para todos los hogares como a través de los quintiles de ingresos.

Por otro lado, los gastos relativos en el grupo de 'leche y productos lácteos' se mantuvieron prácticamente iguales durante el período; sin embargo, este crecimiento fue desigual en los quintiles de ingresos: por ejemplo, los hogares del 
primer quintil de ingresos (Q1) disminuyeron sus gastos alimentarios relativos hasta en un $17 \%$, mientras que los hogares del cuarto quintil de ingresos (Q4) aumentaron sus gastos alimentarios relativos en más del $10 \%$ el período.

\section{Consumo Aparente}

La Tabla 3 presenta los resultados del análisis del consumo aparente de productos lácteos a lo largo del tiempo, para todos los hogares y entre los quintiles de ingresos, así como las áreas rurales/urbanas. Observamos que, si bien el consumo de productos lácteos aumentó entre 1997 y 2012 para la muestra en su conjunto, en realidad experimentó una disminución en el Q1, un aumento marginal en el Q2, y aumentos importantes en los demás quintiles (Q3-Q5).

Tabla 2. Cambios en los gastos relativos en grupos de alimentos para todos los hogares y por quintiles de ingresos (en \%).

\begin{tabular}{lcccccc}
\hline Grupo de alimentos & $\Delta \mathbf{T H}$ & $\Delta \mathbf{Q 1}$ & $\Delta \mathbf{Q} 2$ & $\Delta \mathbf{Q 3}$ & $\Delta \mathbf{Q 4}$ & $\Delta \mathbf{Q 5}$ \\
\hline Cereales & 1.6 & 17.7 & 0.0 & 0.7 & 0.5 & -8.2 \\
Hortalizas & -21.3 & -18.7 & -15.5 & -21.6 & -19.1 & -30.0 \\
\hline Frutas & -18.8 & -31.6 & -10.8 & -7.8 & -18.2 & -23.5 \\
Carne & 8.5 & 2.1 & 8.9 & 8.4 & 9.0 & 9.3 \\
\hline Huevos & -30.5 & -48.9 & -27.3 & -28.8 & -20.4 & -32.5 \\
Leche y productos lácteos & 1.2 & -17.0 & -5.3 & 2.0 & 10.1 & 4.5 \\
\hline Aceites y grasas & -21.5 & -2.2 & -31.2 & -34.0 & -19.6 & -16.0 \\
Dulces & 31.7 & 40.5 & 18.4 & 34.7 & 28.4 & 35.9 \\
\hline Bebidas no alcoholicas & 4.0 & 0.0 & 1.1 & 0.9 & 4.9 & 7.7 \\
Bebidas alcohólicas & -13.8 & -37.7 & 11.8 & -1.9 & -33.0 & -3.1 \\
\hline Especias y condimentos & -15.1 & -6.3 & -16.4 & -23.7 & -15.3 & -13.2 \\
Otros alimentos & 175.6 & 429.7 & 251.9 & 156.8 & 94.0 & 157.1 \\
\hline Comidas preparadas y FDH & 61.1 & 30.0 & 95.9 & 89.1 & 6.9 & 98.6 \\
\hline
\end{tabular}

Tabla 3. Consumo aparente de productos lácteos, por quintiles de ingresos y por área (cc/per cápita/día)

\begin{tabular}{|c|c|c|c|c|c|c|c|c|c|c|c|}
\hline & \multicolumn{3}{|c|}{ Todos Hogares } & \multicolumn{3}{|c|}{ Hogares Urbanos } & \multicolumn{3}{|c|}{ Hogares Rurales } & \multicolumn{2}{|c|}{ Diff Rural-Urbana } \\
\hline & $1997-98$ & 2011-12 & $\Delta(\%)$ & $1997-98$ & $011-12$ & $\Delta(\%)$ & $1997-98$ & $2011-12$ & $2 \Delta(\%)$ & $1997-98$ & 2011-12 \\
\hline Q1 & 253 & 229 & -9.5 & 294 & 280 & -4.8 & 171 & 189 & 10.7 & 71.9 & 47.9 \\
\hline Q2 & 297 & 315 & 6.1 & 345 & 342 & -0.9 & 213 & 267 & 25.4 & 62.0 & 28.0 \\
\hline Q3 & 333 & 380 & 14.1 & 394 & 404 & 2.5 & 230 & 318 & 38.1 & 71.3 & 27.1 \\
\hline Q4 & 402 & 477 & 18.6 & 480 & 501 & 4.4 & 303 & 388 & 27.9 & 58.4 & 29.3 \\
\hline Q5 & 518 & 613 & 18.4 & 610 & 634 & 3.9 & 394 & 490 & 24.3 & 54.8 & 29.4 \\
\hline TH & 365 & 397 & 8.7 & 423 & 448 & 6.0 & 273 & 283 & 3.8 & 54.9 & 58.3 \\
\hline
\end{tabular}

El panorama general es muy diferente cuando se enfoca por separado en áreas rurales y urbanas; aunque las áreas urbanas consumieron en promedio $54.9 \%$ (58.3\%) más que las áreas rurales durante 1997-
98 y 2011-12, las áreas urbanas experimentaron un aumento marginal en el consumo diario en la mayoría de los quintiles de ingresos, mientras que las áreas rurales han visto un aumento importante en el consumo diario en todos los quintiles de ingreso. Estos valores resultaron en una disminución importante del diferencial rural-urbano a lo largo de los quintiles de ingreso durante el período bajo estudio.

Como se señaló en la introducción, el análisis anterior solo incluye artículos alimenticios que fueron comprados por el hogar. Sin embargo, las encuestas de hogares EIH 1997-98 y EIG 2011-12 también brindan información sobre alimentos producidos por los hogares (autoconsumo). A partir de ahora, nuestro análisis incluirá también estos alimentos.

La Tabla 4 presenta los resultados del análisis del consumo aparente de productos lácteos a lo largo del tiempo, incluidos los productos lácteos de producción propia. Es de destacar que, si bien la auto-producción apenas aumentó el consumo aparente de productos lácteos en los hogares urbanos, aumentó en un 56\% en 1997-98 (43\% en 2011-12) en los hogares rurales. Este aumento es más evidente en los hogares rurales Q1 y Q2, con un aumento de $84 \%$ y $72 \%$ en el consumo aparente en $1997-98$ y un aumento de $58 \%$ y $51 \%$ en el consumo aparente en 2011-12.

Tabla 4. Consumo aparente de productos lácteos (incluyendo auto-producción), por quintiles de ingresos y por área (cc/per cápita/día)

\begin{tabular}{|c|c|c|c|c|c|c|c|c|c|c|c|}
\hline & \multicolumn{3}{|c|}{ Todos Hogares } & \multicolumn{3}{|c|}{ Hogares Urbanos } & \multicolumn{3}{|c|}{ Hogares Rurales } & \multicolumn{2}{|c|}{ Diff Rural-Urbana } \\
\hline & $1997-98$ & 2011-12 & $\Delta(\%)$ & $1997-98$ & $2011-12$ & $2 \Delta(\%)$ & $1997-98$ & 2011-12 & $2 \Delta(\%)$ & $1997-9$ & $2011-12$ \\
\hline Q1 & 304 & 295 & -3.1 & 297 & 286 & -3.7 & 314 & 299 & -4.7 & -5.4 & -4.4 \\
\hline Q2 & 362 & 373 & 3.0 & 358 & 350 & -2.2 & 367 & 403 & 9.8 & -2.5 & -13.1 \\
\hline Q3 & 398 & 428 & 7.4 & 413 & 414 & 0.2 & 382 & 455 & 19.2 & 8.1 & -9.1 \\
\hline Q4 & 480 & 510 & 6.3 & 494 & 509 & 3.0 & 465 & 515 & 10.7 & 6.2 & -1.1 \\
\hline Q5 & 596 & 635 & 6.5 & 624 & 638 & 2.2 & 568 & 622 & 9.5 & 9.9 & 2.5 \\
\hline $\mathrm{TH}$ & 431 & 437 & 1.3 & 436 & 456 & 4.6 & 426 & 405 & -4.9 & 2.3 & 12.5 \\
\hline
\end{tabular}

La Tabla 5 presenta los resultados del análisis del consumo aparente de grupos de productos lácteos individuales por quintiles de ingresos y áreas rurales/urbanas. Los resultados muestran una disminución importante en el consumo aparente de leche en polvo en todos los quintiles de ingreso y un aumento notable en el consumo de queso y yogures durante el período. 
Tabla 5. Consumo aparente de productos lácteos (incluyendo auto-producción), por quintiles de ingresos y por área (cc/per cápita/día)

\begin{tabular}{|c|c|c|c|c|c|c|c|c|c|c|c|c|}
\hline & \multicolumn{3}{|c|}{ Todos Hogares } & \multicolumn{3}{|c|}{ Hogares Urbanos } & \multicolumn{3}{|c|}{ Hogares Rurales } & \multicolumn{3}{|c|}{ Diff Rural-Urbana } \\
\hline & $1997-98$ & 2011-12 & $2 \Delta(\%)$ & $1997-98$ & 2011-12 & $\Delta(\%)$ & $1997-98$ & 2011-12 & $2 \Delta(\%)$ & $1997-9$ & 2011-1 & $2 \Delta(\%)$ \\
\hline Q1 & 207 & 182 & -11.9 & 20 & 8 & -59.9 & 64 & 85 & 33.2 & 13 & 19 & 44.1 \\
\hline Urbano & o 196 & 177 & -9.6 & 29 & 13 & -54.8 & 51 & 64 & 24.6 & 21 & 33 & 52.1 \\
\hline Rural & 222 & 185 & -16.5 & 9 & 5 & -38.6 & 81 & 97 & 20.0 & 2 & 11 & 370.8 \\
\hline Q2 & 243 & 231 & -4.8 & 26 & 9 & -65.0 & 76 & 103 & 35.1 & 17 & 30 & 78.2 \\
\hline Urbano & o 237 & 211 & -11.2 & 32 & 11 & -64.4 & 60 & 89 & 49.2 & 29 & 39 & 35.9 \\
\hline Rural & 250 & 258 & 3.3 & 18 & 6 & -67.7 & 96 & 121 & 26.0 & 2 & 18 & 668.2 \\
\hline Q3 & 262 & 265 & 1.2 & 26 & 11 & -56.9 & 93 & 113 & 21.8 & 18 & 39 & 114.8 \\
\hline Urbano & o 266 & 248 & -6.6 & 38 & 12 & -67.7 & 80 & 109 & 35.8 & 29 & 44 & 53.2 \\
\hline Rural & 258 & 300 & 16.0 & 12 & 9 & -27.2 & 106 & 120 & 12.9 & 6 & 27 & 380.3 \\
\hline Q4 & 299 & 280 & -6.2 & 9 & 26 & -24.2 & 116 & 148 & 27.9 & 30 & 56 & 85.0 \\
\hline Urbano & o 288 & 270 & -6.1 & 26 & 29 & -38.2 & 117 & 149 & 27.1 & 43 & 61 & 42.5 \\
\hline Rural & 310 & 310 & 0.2 & 19 & 19 & -17.4 & 115 & 146 & 27.0 & 17 & 39 & 125.2 \\
\hline Q5 & 348 & 326 & -6.3 & 36 & 36 & -33.4 & 155 & 210 & 35.7 & 39 & 62 & 58.4 \\
\hline Urbano & o 320 & 325 & 1.8 & 40 & 40 & -49.2 & 167 & 206 & 23.4 & 58 & 66 & 12.9 \\
\hline Rural & 377 & 332 & -12.1 & 14 & 14 & -51.6 & 143 & 233 & 62.6 & 20 & 44 & 120.6 \\
\hline TH & 273 & 253 & -7.6 & 17 & 17 & -47.5 & 102 & 128 & 26.0 & 24 & 40 & 67.1 \\
\hline Urbano & o 261 & 254 & -2.8 & 22 & 22 & -50.3 & 94 & 130 & 38.1 & 36 & 50 & 40.6 \\
\hline Rural & 287 & 251 & -12.6 & 8 & 8 & -54.6 & 110 & 124 & 12.5 & 10 & 22 & 115.9 \\
\hline
\end{tabular}

La Tabla 6 presenta los resultados del análisis del consumo aparente de calcio a lo largo del tiempo, para todos los hogares y entre los quintiles de ingresos, así como las áreas urbanas y rurales. Se observó que el consumo aparente de calcio aumentó marginalmente durante el período 1997-2012 (2.3\%); sin embargo, el quintil inferior de los hogares urbanos y rurales experimentó una disminución marginal en el consumo aparente de calcio.

Tabla 6. Consumo aparente de calcio (incluyendo la auto-producción) por quintiles de ingreso y área (mg/per cápita/día)

\begin{tabular}{|c|c|c|c|c|c|c|c|c|c|c|c|}
\hline & \multicolumn{3}{|c|}{ Todos Hogares } & \multicolumn{3}{|c|}{ Hogares Urbanos } & \multicolumn{3}{|c|}{ Hogares Rurales } & \multicolumn{2}{|c|}{ Diff Rural-Urban } \\
\hline & $1997-98$ & $2011-1$ & $\Delta(\%)$ & $1997-98$ & $2011-1$ & $2 \Delta(\%)$ & $1997-98$ & $2011-1$ & $2 \Delta(\%)$ & $1997-9$ & $2011-12$ \\
\hline Q1 & 355 & 346 & -2.6 & 346 & 338 & -2.4 & 368 & 351 & -4.6 & -6.1 & -3.9 \\
\hline Q2 & 422 & 440 & 4.2 & 418 & 414 & -1.0 & 427 & 474 & 11.0 & -2.3 & -12.8 \\
\hline Q3 & 465 & 505 & 8.6 & 481 & 489 & 1.7 & 447 & 538 & 20.2 & 7.4 & -9.1 \\
\hline Q4 & 559 & 599 & 7.0 & 574 & 597 & 3.9 & 544 & 605 & 11.1 & 5.5 & -1.3 \\
\hline Q5 & 692 & 741 & 7.2 & 718 & 744 & 3.5 & 665 & 729 & 9.7 & 8.1 & 2.0 \\
\hline $\mathrm{TH}$ & 502 & 514 & 2.3 & 506 & 536 & 5.9 & 498 & 477 & -4.4 & 1.5 & 12.4 \\
\hline
\end{tabular}

\section{DISCUSIÓN}

Este estudio analiza los cambios en los gastos relativos y el consumo aparente de productos lácteos y calcio en hogares paraguayos con niños menores de 19 años durante el período comprendido entre 1997 y 2012, tanto para todos los hogares como para los quintiles de ingresos, así como por zonas rurales y urbanas.
El análisis muestra que, incluyendo la autoproducción, el consumo aparente de productos lácteos en los hogares paraguayos con niños menores de 19 años fue de 437 cc/per cápita/día en 2011-12, solo un poco más de $431 \mathrm{cc} /$ per cápita/día observado en 1997-98. Esto es considerablemente inferior a los $600 \mathrm{cc}$ de productos lácteos por persona por día recomendados en las últimas Guías Alimentarias del Paraguay ${ }^{(7)}$.

Sin embargo, el consumo aparente de productos lácteos varió sustancialmente entre los quintiles de ingresos, aumentando de un promedio de $295 \mathrm{cc} /$ per cápita/día en los hogares del Q1 a un promedio de $635 \mathrm{cc} /$ per cápita/día en los hogares del Q5. Solo los hogares en el quintil superior de ingresos (Q5) alcanzaron la recomendación de $600 \mathrm{cc}$ de productos lácteos por persona por día. El consumo aparente aumentó marginalmente entre 1997 y 2012 a través de los quintiles de ingreso, con la excepción del Q1 que experimentó una disminución marginal (-3.1\%) durante el período.

Los resultados resaltan la importancia de la autoproducción para la ingesta de productos lácteos, especialmente en áreas rurales. De hecho, si bien la auto-producción apenas aumentó el consumo aparente de productos lácteos en los hogares urbanos, aumentó en un 56\% en 1997-98 y en un $43 \%$ en $2011-12$ en los hogares rurales. La diferencia entre el consumo aparente de productos lácteos de hogares rurales y urbanos diferencial rural-urbano experimentó un deterioro de 12.5\% para la muestra como un todo durante el período 1997-2012.

Varios hallazgos se destacan al analizar el consumo aparente de productos lácteos por grupo de productos. En primer lugar, el consumo aparente de leche disminuyó en el período 1997-2012 (-7,6\%), especialmente en las zonas rurales $(-12,6 \%)$. En segundo lugar, el consumo aparente de yogur aumentó en un $67 \%$, impulsado principalmente por los aumentos en los hogares rurales Q1-Q3. Y, por último, el consumo aparente de queso aumentó en un $26 \%$, impulsado principalmente por los aumentos en los hogares urbanos Q1-Q3. En relación con el consumo aparente total de productos lácteos, la importancia relativa de la leche disminuyó, 
mientras que la importancia relativa del queso y el yogur aumentó durante el período.

Vale la pena señalar que el consumo aparente de productos lácteos en 2011-12 fue significativamente mayor que la disponibilidad de leche en Paraguay en ese momento. Según las hojas anuales de balance de alimentos de la FAO para Paraguay, que ofrecen una visión completa del suministro de leche (o de la disponibilidad de leche) en el país ${ }^{(8)}$, la cantidad de suministro de leche por persona era de $243 \mathrm{cc}$ per cápita/día en 1997-98. y 212 ccper cápita/día en 201112, por debajo incluso del consumo aparente de leche (sin otros productos lácteos) durante el período (273 cc y 253 cc per cápita/día durante 199798 y 2011-12, respectivamente).

En términos del consumo de calcio, este alcanzó un promedio de $514 \mathrm{mg} /$ per cápita/día para la muestra como un todo, mientras que una vez más muestra diferencias importantes entre los quintiles individuales de ingreso. En particular, el consumo de calcio fue dos veces más alto en Q5 en comparación con los hogares Q1: $741 \mathrm{mg}$ per cápita/día en Q5 frente a $346 \mathrm{mg} /$ per cápita/día en Q1. Es decir, solo los hogares en el quintil superior de ingresos (Q5) alcanzaron la recomendación de 678mgper cápita/día, derivado de las tres porciones de $200 \mathrm{ml}$ de leche entera recomendadas por las últimas Guías Alimentarias del Paraguay ${ }^{(7)}$ y equivalentes a aproximadamente $68 \%$ de las recomendaciones dietéticas diarias $(1.000 \mathrm{mg}$ de calcio) para una persona adulta (hombres entre $25 \mathrm{y}$ 65 años y mujeres entre 25 y 50 años) ${ }^{(9)}$.

Es importante recalcar que los valores promedio de consumo de calcio son solo valores promedio que no consideran ni la edad ni la distribución por sexo de los miembros del hogar. Por lo tanto, es muy posible que las recomendaciones dietéticas diarias se cumplan en muchos hogares con niños pequeños, cuyas necesidades diarias combinadas de calcio son mucho más bajas que si se aplicaran a un grupo de personas adultas. Con este fin, un estudio reciente que examinó los patrones de consumo de fluidos entre 2.352 niños y adolescentes en los departamentos de Asunción y Central y Caaguazú encontró que los niños menores de 10 años consumían en promedio $395 \mathrm{ml}$ de leche y productos derivados por día, niños con edades comprendidas entre 10 y 13 años $383 \mathrm{ml}$ de leche y productos derivados por día, y niños de 14 años en adelante 865 $\mathrm{ml}$ de leche y productos derivados por día ${ }^{(10)}$.

Es importante mencionar algunas limitaciones del presente estudio. Por un lado, el estudio no consideró productos lácteos consumidos fuera del hogar debido a que esta información no se encuentra disponible. Esto puede ocasionar una subestimación del verdadero consumo de los productos lácteos. Por otro lado, los datos de compra consideran la disponibilidad de alimentos en lugar del consumo de alimentos; no todos los alimentos disponibles para el hogar son consumidos por los miembros del hogar. Algunos alimentos se pueden echar a perder, perder o desperdiciar, se les da a los animales o se sirven a personas que no son miembros del hogar ${ }^{(11)}$. En este caso, las estimaciones del consumo aparente pueden sobreestimar el verdadero consumo de los productos lácteos.

\section{CONCLUSIÓN}

Para la mayoría de los hogares paraguayos con niños menores de 19 años y, en particular, aquellos en los cuatro quintiles de ingresos más bajos, el consumo aparente de productos lácteos fue considerablemente menor que las cantidades recomendadas en las guías alimentarias. Hallazgos similares son válidos para el consumo aparente de calcio. Aunque no se encontraron diferencias sustanciales entre los hogares rurales y urbanos, los hallazgos resaltan la importancia de la autoproducción para este resultado.

La recomendación de consumir más porciones de lácteos tiene como objetivo generar múltiples beneficios nutricionales y de salud. Estos incluyen no solo un mayor consumo de calcio, vitamina D, potasio y otros nutrientes que a menudo se consumen por debajo de los niveles recomendados, sino también importantes beneficios para la salud, como una mejor salud ósea y un menor riesgo de enfermedad cardiovascular y diabetes tipo $\mathrm{II}^{(3)}$. El presente estudio destaca la necesidad de promover el consumo de productos lácteos en hogares de ingresos medios a bajos para que puedan cosechar dichos beneficios. 


\section{AGRADECIMIENTOS}

Este estudio fue realizado en el marco del proyecto “La transición nutricional en el Paraguay: en donde estamos" (PINV 15-1304), el cual es ejecutado por el Instituto Desarrollo y financiado por el Consejo

\section{REFERENCIAS}

1. WHO. Diet, nutrition and the prevention of chronic diseases: report of a joint WHO/FAO expert consultation. Geneva: WHO; 2016. (WHO Technical Report Series Nr. 916).

2. Rozenberg S, Body JJ, Bruyére O, Bergmann P, Brandi $\mathrm{ML}$, Cooper $\mathrm{C}$, et al. Effects of dairy products consumption on health: Benefits and beliefs - A commentary from the Belgian Bone Club and the European Society for Clinical and Economic Aspects of Osteoporosis, Osteoarthritis and Musculoskeletal Diseases. Calcif Tissue Int. 2016; 98:1-17. doi: 10.1007/s00223-015-0062-x.

3. Quann EE, Fulgoni VL, Auestad N. Consuming the daily recommended amounts of dairy products would reduce the prevalence of inadequate micronutrient intakes in the United States: diet modeling study based on NHANES 2007-2010. Nutrition Journal. 2015; 14:90. doi:10.1186/s12937-015-0057-5.

4. Heaney RP. Dairy and bone health. J Am Coll Nutr. 2009;28(Suppl 1):82S-90S.

5. FAO. Milk and dairy products in human nutrition: Questions and Answers. Food and Agriculture Organization; 2012.

6. Crovetto MM, Uauy R. Cambios en el consumo aparente
Nacional de Ciencias y Tecnología (CONACYT) del Paraguay, a través del programa PROCIENCIA con recursos del Fondo para la Excelencia de la Educación e Investigación (FEEI) del Fondo Nacional de Inversión Pública y Desarrollo (FONACIDE).

de lácteos, bebidas azucaradas y jugos procesados en el Gran Santiago. Chile. 1987-2007. Rev. Méd. Chile. 2014; 142(12):1530-1539.

7. MSPyBS. Instituto Nacional de Alimentación y Nutrición. Guías Alimentarias del Paraguay. 2da Actualización. Comité técnico Nacional de Elaboración e Implementación de la Guías Alimentarias; 2015.

8. FAO. Food Balance Sheets. (Internet). 2016. (Citado el 12 de marzo 2018). Recuperado a partir de: http://www.fao.org/faostat/en/\#data/FBS.

9. Institute of Medicine. Dietary reference intakes for vitamin D and calcium; Ross AC, Taylor CL, Yaktine AL, et al., eds. Washington D.C: The National Academies Press; 2011.

10. Ríos P, Aguilar G, Estigarribia G, Sanabria M, Sanabria G, Kawabata A, et al. Patrones de ingesta de líquidos: un estudio epidemiológico en niños y adolescentes escolarizados de Paraguay. Revista Científica Estudios E Investigaciones. 2017; (6). doi: 10.26885/rcei.foro.2017.102.

11. Fiedler JL, Mwangi DM. Using household consumption and expenditure surveys to make inferences about food consumption, nutrient intakes, and nutrient status. IFPRI Discussion Paper 01571. Washington, D.C: International Food Policy Research Institute; 2016. 